\title{
Correction
}

\section{A 12-year retrospective audit study of tooth loss in a general dental practice (BDJ 189:2, 22 July 2000)}

Dr Clive Nicholls has notified us of an error in his paper. On page 99 in the results section, the sentence that reads:

'on ly 21 patients $(13 \%)$ had probing depths of $5 \mathrm{~mm}$ or greater at initial examination',

Should read:

'only 33 patients $(21 \%)$ had probing depths of $7 \mathrm{~mm}$ or greater at initial examination'. 